\title{
A COMPARISON OF VITAMIN A AND LEUCOVORIN FOR THE PREVENTION OF METHOTREXATE- INDUCED MICRONUCLEI PRODUCTION IN RAT BONE MARROW
}

\author{
Sampath Madhyastha, Latha V Prabhu, Saralaya V, Rajalakshmi Rai
}

doi: 10.1590/S1807-59322008000600019

\begin{abstract}
Madhyastha S, Prabhu LV, Saralaya V, Rai R. A comparison of vitamin A and leucovorin for the prevention of methotrexateinduced micronuclei production in rat bone marrow. Clinics. 2008;63:821-6.
\end{abstract}

INTRODUCTION: Methotrexate, a folate antagonist, is a mainstay treatment for childhood acute lymphoblastic leukemia. It is also widely used in a low dose formulation to treat patients with rheumatoid arthritis. In rats, methotrexate is known to induce micronuclei formation, leading to genetic damage, while vitamin A is known to protect against such methotrexate-induced genetic damage. Leucovorin (folinic acid) is generally administered with methotrexate to decrease methotrexate-induced toxicity.

OBJECTIVES: We aimed to determine whether vitamin A and leucovorin differed in their capacity to prevent formation of methotrexate-induced micronuclei in rat bone marrow erythrocytes. The present study also aimed to evaluate the effect of combined treatment with vitamin A and leucovorin on the formation of methotrexate-induced micronuclei.

METHODS: Male and female Wistar rats $(\mathrm{n}=8)$ were injected with $20 \mathrm{mg} / \mathrm{kg}$ methotrexate (single i.p. dose). The control group received an equal volume of distilled water. The third and fourth groups of rats received vitamin A (5000 IU daily dose for 4 successive days) and leucovorin $(0.5 \mathrm{mg} / \mathrm{kg}$ i.p. dose for 4 successive days), respectively. The fifth and sixth groups of rats received a combination of vitamin A and a single dose of methotrexate and a combination of leucovorin and methotrexate, respectively. The last group of rats received a combination of leucovorin, vitamin A and single dose of methotrexate. Samples were collected at 24 hours after the last dose of the treatment into 5\% bovine albumin. Smears were obtained and stained with May-Grunwald and Giemsa. One thousand polychromatic erythrocytes were counted per animal for the presence of micronuclei and the percentage of polychromatic erythrocyte was determined.

RESULTS: Comparison of methotrexate-treated rats with the control group showed a significant increase in the percentage of cells with micronuclei and a significant decrease polychromatic erythrocyte percentage. Combined methotrexate and vitamin A therapy and combined methotrexate and leucovorin therapy led to significant decreases in the micronuclei percentage and an increase in polychromatic erythrocyte percentage when compared to rats treated with methotrexate alone. Leucovorin was found to be more effective than vitamin A against the formation of methotrexate-induced micronuclei.

CONCLUSIONS: Both vitamin A and leucovorin provided significant protection against genetic damage induced by methotrexate.

KEYWORDS: Micronuclei; Rat bone marrow erythrocytes; Methotrexate; Vitamin A; Leucovorin.

\section{INTRODUCTION}

Methotrexate, an inhibitor of dihydrofolate reductase, is known to induce micronuclei formation upon multiple

Department of Anatomy, Kasturba Medical College - Mangalore, India. Email: madhyast1971@gmail.com

Tel.: 918242423452

Received for publication on July 26, 2008

Accepted for publication on September 4, 2008 doses. ${ }^{1}$ Methotrexate is a mainstay treatment for childhood acute lymphoblastic leukemia with meningeal infiltration and also for many other forms of carcinoma. Methotrexate has also become an important therapeutic alternative for the treatment of severe psoriasis ${ }^{2}$ as methotrexate has antiinflammatory properties. More specifically, it reduces lymphocyte proliferation, rheumatoid factor production and leukocyte interactions. It is used intermittently at a low dosage to induce remission of refractory rheumatoid 
arthritis. ${ }^{3}$ Methotrexate is also used in the management of ectopic pregnancy. ${ }^{4,5}$ Methotrexate restricts the synthesis of thymidylate and purine nucleotides by inhibiting dihydrofolate reductase and, to a lesser extent, thymidylate synthatase. In cells treated with methotrexate, a progressive accumulation of strand breaks in mature DNA (post-replicated DNA) was detected by Li and Kaminskas. ${ }^{6}$ They postulated that the strand brake arose from spontaneous and normally repaired DNA lesions that were not repaired due to a shortage of dTTP and purine nucleotides. The cytogenetic effect of methotrexate has been studied in mouse tumors, ${ }^{7}$ cultured potorous cells ${ }^{8}$ and human cells in vivo. ${ }^{9}$ Methotrexate was found to be a clastogenic agent in tumor cells and in cultured mammalian cells. A collaborative study ${ }^{10}$ on various chemicals for micronuclei formation revealed that only methotrexate had a positive response in a single dose.

Many reports ${ }^{11,12}$ suggest that vitamin A protects against micronuclei induction. In an earlier study, ${ }^{13}$ rats treated with a combination of methotrexate and vitamin $\mathrm{A}$ had a significantly reduced frequency of micronuclei formation when compared to methotrexate $(20 \mathrm{mg} / \mathrm{kg})$-treated rats.

Leucovorin is a form of folic acid. Folic acid is a naturally occurring substance that is important for the formation of red and white blood cells. Folic acid is present in many foods, such as beans, peas, oranges, spinach. Leucovorin is used to reduce the side effects of large doses of medications, such as methotrexate (Rheumatrex), pyrimethamine (Daraprim), trimethoprim (Trimpex, Proloprim), that may reduce the effects of folic acid in the body. In the present study, we planned to compare the efficacy of vitamin A with that of leucovorin with respect to their ability to inhibit formation of methotrexate-induced micronuclei. We also planned to evaluate the effects of drug combinations on methotrexate-induced micronuclei in the bone marrow of rats.

\section{MATERIALS \& METHODS}

Animals: Male and female adult Wistar rats weighing approximately $150-170(158.5 \pm 6.34) \mathrm{g}$ were used in this study. Animals were selected on the basis of adequate body weight gain and the lack of any clinical signs of disease or injury. All animals were identified by tail marking with indelible ink, which lasted for the duration of the study. The animals were individually weighed and randomly assigned to one of seven groups so that there were no statistically significant differences among group body weight means. Each group consisted of eight animals (four males and four females). Animals were maintained under controlled light (10h light: $14 \mathrm{~h}$ dark), temperature $\left(22 \pm 3^{\circ} \mathrm{C}\right)$, and humidity (approximately $50 \pm 10 \%$ ) conditions in an air-conditioned animal house. All rats were maintained on standard rat food and water ad libitum. The experimental procedure was approved by the institutional ethics committee. Breeding and maintenance of the animals were in accordance with the guidelines of the Government of India for the use of laboratory animals.

\section{Animal groups}

Group 1: Rats were given an equivalent volume of distilled water.

Group 2: Rats received $20 \mathrm{mg} / \mathrm{kg}$ body weight dose of methotrexate (single i.p. dose)

Group 3: Rats received vitamin A (5000 IU for four successive days)

Group 4: Rats received leucovorin $(0.5 \mathrm{mg} / \mathrm{kg}$ for four successive days)

Group 5: Rats received methotrexate $(20 \mathrm{mg} / \mathrm{kg}$, single, i.p. dose) and vitamin A (5000 IU for four successive days before the administration of methotrexate)

Group 6: Rats received methotrexate $(20 \mathrm{mg} / \mathrm{kg}$, single i.p. dose) and leucovorin $(0.5 \mathrm{mg} / \mathrm{kg}$ for four successive days before the administration of methotrexate)

Group 7: Rats received methotrexate $(20 \mathrm{mg} / \mathrm{kg}$, single i.p. dose) along with vitamin A (5000 IU for four successive days) and leucovorin $(0.5 \mathrm{mg} / \mathrm{kg}$ for four successive days) before the administration of methotrexate.

\section{Chemicals}

Methotrexate, vitamin A and leucovorin (calcium leucovorin) were obtained from Biochem Pharmaceutical Industries (Ahmedabad, India). Bovine albumin (Sigma grade, 96-99\% B No.140), May-Gruenwald powder and Giemsa powder were obtained from Romoli Co., (Mumbai). All other chemicals and reagents were of HPLC or analytical grade (Sigma, St. Louis, Mo.).

\section{Micronuclei test}

In the present study, Schmid's ${ }^{14}$ standard procedure was followed with slight modifications. Instead of foetal calf serum, 5\% bovine albumin was used as suspending medium to collect the bone marrow ${ }^{15}$. The rats were sacrificed $24 \mathrm{~h}$ after the last methotrexate treatment, femurs were trimmed, and a blunt needle was pushed to pierce the marrow cavity. The marrow was flushed through a syringe by $5 \%$ bovine albumin to obtain a fine suspension. The suspension was centrifuged at $1000 \mathrm{rpm}$ for 8 to 10 minutes. The supernatant was discarded and half of a drop of fresh suspending medium was added and mixed thoroughly by a Pasteur pipette. A small drop of 
suspension was placed on one end of the slide and a smear was prepared (3-4 slides/animal). Slides were cooled and air dried overnight, fixed by methanol for 5 minutes, dried and stained with May-Grunwald and then with a combination of May-Grunwald and phosphate buffer, $\mathrm{pH} 6.8$, for the proper color differentiation of polychromatic erythrocytes (PCEs) and normochromatic erythrocytes (NCEs). Finally, slides were stained with Geimsa and phosphate buffer, $\mathrm{pH}$ 6.8, for micronuclei staining. After washing with distilled water and phosphate buffer, slides were dried and mounted. One thousand PCEs were screened per animal and micronucleated PCEs (MNPCEs) were quantified. Consequently, identified NCEs and micronucleated NCEs (MNNCEs) were quantified. The percentages of MNPCE and PCE were calculated for each animal.

\section{Statistical analysis}

The data are expressed as mean $\pm \mathrm{SD}$. The significance of differences among the groups were assessed using an one way analysis of variance (ANOVA) test followed by Bonferroni's multiple comparison test. $\mathrm{P}$ values $<0.05$ are considered as significant. Differences in the data for the male and female groups were assessed by the Mann-Whitney unpaired $t$ test.

\section{RESULTS}

Sex-related differences were not observed with respect to either micronuclei induction or PCE\%; therefore, data from males and females were collapsed into one group. Methotrexate induced a significantly greater $(\mathrm{p}<0.001)$ number of micronuclei when compared to the control (group 1), vitamin A-treated (group 3), or leucovorin-treated groups (group 4). There was not a significant difference ( $>0.05$ ) between control and vitamin A (group 3)-treated or leucovorin (group 4)-treated rats. The micronuclei percentage was significantly reduced $(\mathrm{p}<0.001)$ in the combined methotrexate and vitamin A (group 5) treatment group as well as in the combined methotrexate and leucovorin (group 6) treatment group when compared to the methotrexate (group 2)-treated rats. Combined treatment with vitamin $\mathrm{A}$ and leucovorin with methotrexate (group 7 ) did not show any significant difference $(p>0.05)$ when compared to the combined methotrexate and vitamin A (group 5) or methotrexate and leucovorin (group 6) treatment groups. These results indicate that treatment with vitamin A and leucovorin had the same effect as treatment with vitamin A or leucovorin alone. The percentage of cells with induced micronuclei was marginally significantly $(\mathrm{p}<0.05)$ different for the methotrexate and vitamin (group 5) treatment group relative to the methotrexate and leucovorin (group 6) treatment group. This result indicates that leucovorin exerts a greater protective effect than vitamin A against methotrexateinduced micronuclei formation (Figure 1).

The PCE\% differed significantly $(\mathrm{p}<0.01)$ between the methotrexate-treated (group 2) and control (group 1) groups of rats. The PCE\% was significantly increased $(\mathrm{p}<0.05)$ in the group treated with methotrexate and vitamin A (group 5 ) as well as that treated with methotrexate and leucovorin (group 6) when compared with methotrexate-treated rats (group 2) (Figure 2). The PCE\% did not differ between the groups treated with methotrexate and vitamin A (group 5) and methotrexate and leucovorin (group 6).

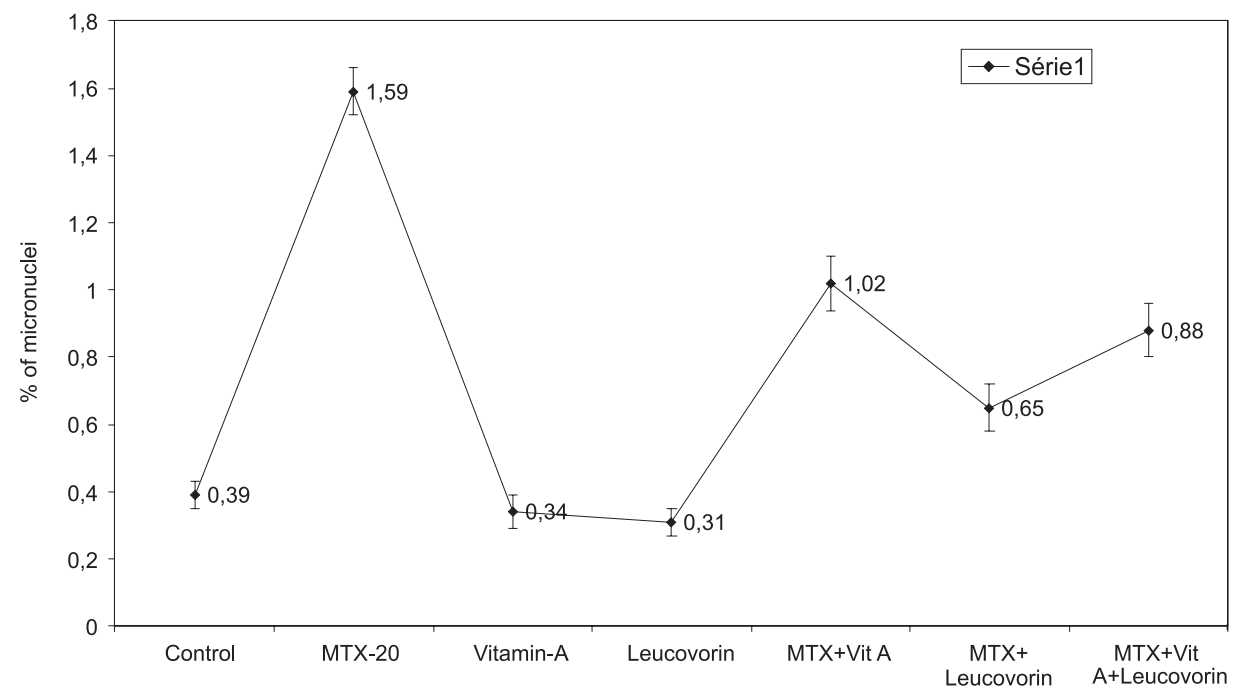

Figure 1 - The percentage of micronuclei-induction in various gropus of rats. Error bar indicates \pm SE $(n=8)$, Anova significance, $F=53.145$ 


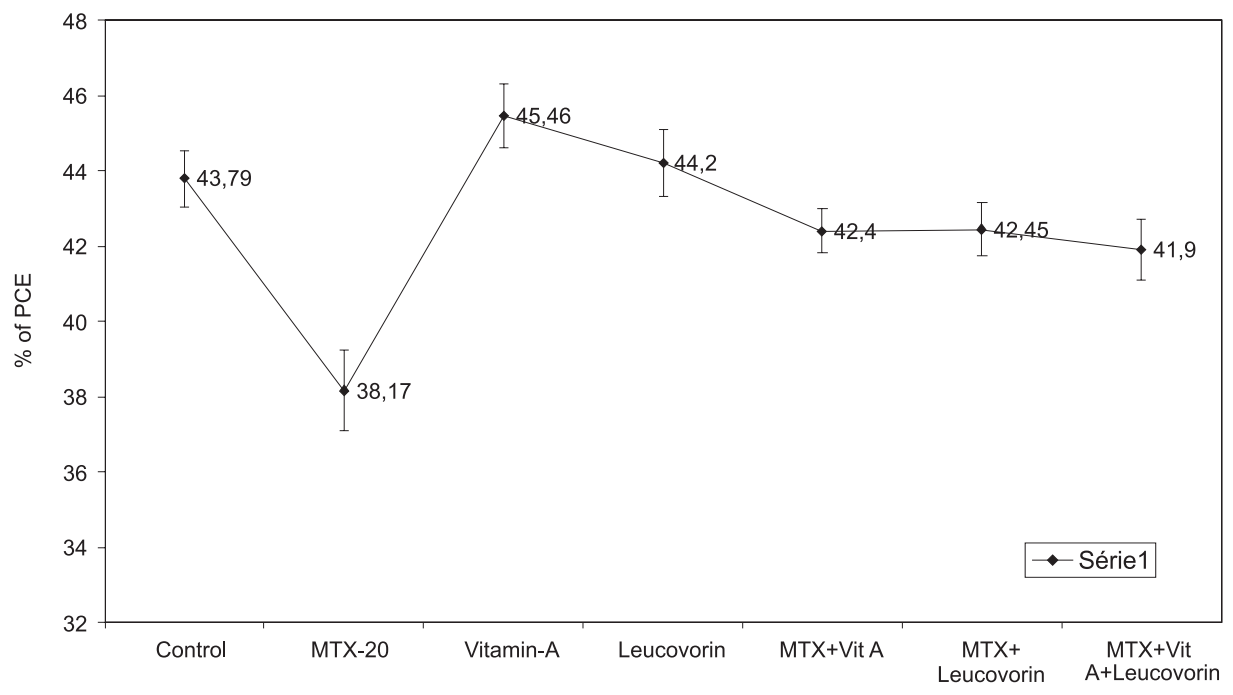

Figure 2 - The percentage of polychromatic erythrocyte in various gropus of rats. Error bar indicates $\pm \mathrm{SE}(\mathrm{n}=8)$, Anova significance, $\mathrm{F}=8.001$

\section{DISCUSSION}

Methotrexate is widely used, in low doses, for the treatment of psoriasis, ${ }^{16}$ autoimmune diseases, inflammatory bowel diseases, asthma, ${ }^{17-20}$ inflammatory myopathy, ${ }^{21}$ and panuveitis. ${ }^{22}$ The clinical utility of methotrexate must be checked against its potential side effects. ${ }^{23}$

People with low and high micronuclei yields show low and high frequencies of chromosome aberration, respectively. ${ }^{24}$ Hence, the micronuclei assay is often used to detect the frequency of chromosomal aberrations. As a number of genetic diseases are characterized by instable chromosomes or by mal-distribution of chromosomes, it is not surprising that also micronucleus frequencies are higher in patients in some of the genetic diseases. Druginduced chromosomal aberrations or the mal distribution of chromosomes can lead to the development of genetic disease. ${ }^{25}$ These chromosomal changes may also lead to the development of tumors. ${ }^{26}$ According to this hypothesis, a patient with a high micronuclei score is prone to chromosome breakage which may lead to tumor formation. Stich et al. ${ }^{27}$ presented evidence which indicated that micronuclei may serve as predictors of carcinogenic risk. This group demonstrated that, after exposure to certain chemicals, tissues that are at elevated risk for cancer show a markedly enhanced number of micronuclei.

In the present study, methotrexate-treated rats had a significantly increased number of micronuclei when compared to a control group of rats. Methotrexate penetrates bone marrow cells and binds to the dihydrofolate reductase enzyme, thereby completely inhibiting its activity. Continuous inhibition of DHFR may cause an imbalance in the deoxynucleotide pools due to shortages in thymidylate and purine nucleotides, which may result in the formation of DNA lesions. ${ }^{6}$ In addition, complete inhibition of DHFR may severely suppress the generation of PCEs from erythroblasts in the bone marrow.

Rats treated with vitamin A and methotrexate had a significantly reduced number of micronuclei when compared to rats treated with methotrexate alone, demonstrating the protective effect of vitamin A. Rats treated with vitamin A alone did not present a significant difference in spontaneous micronuclei formation when compared to control rats, which received distilled water. The PCE\% was increased in rats treated with vitamin A and methotrexate when compared to rats treated with methotrexate alone. Hence, our study confirms that vitamin A minimizes the genotoxic and cytotoxic effects of methotrexate. The protective effect of vitamin A against genotoxicity and cancer chemoprevention has been studied by many groups. Slamenova et al. ${ }^{11}$ observed that dietary intake of vitamin A reduced micronuclei induction in rat hepatocytes challenged with various carcinogens. Similar results were reported by Alaovi-Jamali et al. ${ }^{28}$ Protective effects of vitamin A against micronuclei induction in buccal mucosa of betel nut/ tobacco chewers were also reported. ${ }^{29,30}$ Numerous in vitro experiments have been performed in order to verify the true role played by beta-carotene, a precursor of vitamin A on cell proliferation and differentiation; until now, findings have been very encouraging, uniformly showing that beta-carotene can affect carcinogenesis, particularly in early stages, through an antigenotoxic action. Antioxidant functions, immunomodulatory effects and control of intercellular messages via gap junctions are possible action mechanisms of the ability of beta-carotene to block the carcinogenetic process. ${ }^{31}$ 
The results of the present study clearly demonstrate that leucovorin has a protective effect against methotrexateinduced micronuclei formation in rat bone marrow. The $\mathrm{PCE} \%$ was enhanced in rats treated with leucovorin and methotrexate when compared to rats treated with methotrexate alone. Interestingly, leucovorin-treated rats showed a reduced number of micronuclei following methotrexate treatment when compared to vitamin A-treated rats. Thus, our study clearly demonstrates that leucovorin is more effective than vitamin A against methotrexate-induced micronuclei in rat bone marrow. Our study also indicates that the combination of vitamin A and leucovorin had almost the same effect as vitamin A or leucovorin alone against methotrexate-induced micronuclei formation. Folinic acid (leucovorin) is a 5-formyl derivative of tetrahydrofolic acid. It is readily converted to other reduced folic acid derivatives (e.g. tetrahydrofolate), and thus has vitamin activity which is equivalent to folic acid. However, since it does not require the action of dihydrofolate reductase for its conversion, its function as a vitamin is unaffected by inhibition of this enzyme by drugs such as methotrexate.
Folinic acid, therefore, allows for purine/pyrimidine synthesis to occur in the presence of dihydrofolate reductase inhibition, such that some normal DNA replication and RNA transcription processes can proceed. Folinic acid is administered at the appropriate time following methotrexate as part of a complete chemotherapeutic plan, so that it may "rescue" bone marrow and gastrointestinal mucosa cells from methotrexate. Shahin et al. ${ }^{32}$ demonstrated the protective effects of leucovorin against methotrexate-induced genotoxicity in humans as well as animal models. Similar results were also reported by Chennakeshava et al..$^{33}$

The results of the present study are in accord with previous studies that have shown amelioration of the genotoxic effects of methotrexate by both vitamin A and leucovorin in rats. In addition, the present study claims that leucovorin is more effective at minimizing micronuclei induction in comparison to vitamin A. Interestingly, combined treatment with both vitamin A and leucovorin was not significantly different from treatment with either vitamin A or leucovorin alone with regard to protection from methotrexate-induced micronuceli formation.

\section{REFERENCES}

1. Kasahar Y, Nakai Y, Miura D, Yagi K, Hirabayashi K, Makita T. Mechanism of induction of micronuclei and chromosome aberration of micronuclei and chromosome aberrations in mouse bone marrow by multiple treatments of methotrexate. Mutat Res. 1992;280:117-28.

2. Clark CM, Kirby B, Morris AD, Davison S, Zaki I, Emerson R, et al. Combination treatment with methotrexate and cyclosporine for severe Recalcitrant psoriasis. Br J Dermatol. 1999;141:279-82.

3. Hoffmeister RT. Methotrexate therapy in rheumatoid arthritis: 15 years experience. Am J Med. 1983;75:69-73.

4. Tulandi T, Bret PM, Atri M, Senteman M. Treatment of ectopic pregnancy by transvaginal intratubal methotrexate administration. Obstet Gynecol. 1991;77:627-30.

5. Brown DL. Serial endovaginal sonography of ectopic pregnancies treated with methotrexate. Obset Gynecol. 1991;77:406-9.

6. Li JC and Kaminskas E. Accumulations of DNA strand break and methotrexate cytotoxicity. Proc Natl cad Sci USA. 1984;81:5694-8.

7. Rodriguez Murcia C, Arroyo Nombela J. Cytological aberrations produced by methotrexate in mouse ascities tumours. Mut Res. 1972;14:405-12.

8. Hittelman W. The type and time of occurrence of aminopterineinduced chromosome aberrations in cultured potorous cells. Mut Res. 1973;18:93-102.
9. Jensen M, Nyfors A. Cytogenetic effects of methotrexate on human cells in vivo. Mut Res. 1979;64:339-43.

10. CSGMT/JEMS-MMS. Collaborative study group for the micronucleus test. Single versus multiple dosing in the micronucleus test: the summary of the fourth collaborative study by CSGMT/JEMS-MMS. Mut Res. $1990 ; 234: 205-22$

11. Slamenova D, Chalupa I, Robichova S, Gabelova A, Farkasova T, Hrusovska L, et al. Effect of dietary intake of Vitamin A or E on the level of DNA damage, chromosomal aberrations and micronuclei induced in freshly isolated rat hepatocytes by different carcinogens. Nutr Cancer. 2002;42:117-24.

12. Stich HF, Stich W, Rosin MP, Vallejera MO. Use of the micronucleus test to monitor the effect of vitamin A, beta-carotene and canthaxanthin on the buccal mucosa of betel/tobacco chewers. Int J Cancer. $1984 ; 15: 34: 745-50$.

13. Madhyastha S, Bairy KL \& Somayaji SN. Protective effect of vitamin A on methotrexate induced micronuclei. Iranian Journal of Pharmacology \& Therapeutics. 2007;6:1-4.

14. Schmid W. The micronucleus test for cytogenetic analysis. In: Hallaender A (Ed). Chemical mutagens, principles and methods for their detection, vol.4. Plenum Press: New York. 1976;31-53.

15. Narayan K, D'Souza UJ, Rao KPS. The genotoxic and cytotoxic effects of ribavirin in rat bone marrow. Mutat Res. 2002;521:179-85 
16. Pearce HP, Wilson BB. Erosion of psoriatic plaques: an early sign of methotrexate toxicity. J Am Acad Dermatol. 1996;35:835-8.

17. Alarcon GS. Methotrexate: its use for the treatment of rheumatoid arthritis disorders. In: Koopman WJ (Ed) Arthritis and Allied Conditions Textbook of Rheumatology, $13^{\text {th }}$ ed., Balitmore, Williams and Wilkins. 679-707.

18. Baughman RP. Methotrexate for sarcoidosis, Sarcoidosis Vasc, Diffuse Lung Dis. 1998;15:147-9.

19. Boehm IB, Boehm GA, Bauer R. Management of cutaneous lupus erythematous with low dose methotrexate; indication for modulation for inflammatory mechanisms. Rheumatol Int. 1998;18:59-62.

20. Hirohata S, Suda H, Hashimato T. Low-dose weekly methotrexate for progressive neuropsychiatric manifestations in Behcet's disease. J Neurol Sci. 1998;159:181-5.

21. Levine TD, Pestronk A. Inflammatory myopathy with cytochrome oxidase negative muscle fibres: methotrexate treatment. Muscle Nerve. 1998;21:1724-8.

22. Dev S, Mc Callum RM, Jaffe GJ. Methotrexate treatment for sarcoidassociated panuveitis. Ophthalmology. 1999;106:111-8.

23. Sanoval DM, Alarcon GS. Adverse events in methotrexate-treated rheumatoid arthritis patients. Br J Rheumatol. 1995;34:49-56.

24. Norman A, D Bass, D Roe, Screening human populations for chromosome aberrations. Mutat Res. 1985;143:155-60.

25. Muller Wu and Streffer C. In: Advances in Mutagenenesis research-5.n Edited by Obe G. Springer-Verlag. 1994; pp. 76-8.
26. Sandberg AA, A Chromosomal hypothesis of oncogenesis. Cancer Genet Cytogenet. 1983;8:277-85.

27. Stich HF, Acton AB, Palcic B: Towards an automated micronucleus assay as an internal dosimeter for carcinogenic exposed human population groups. Recent Results Cancer Res. 1990;120:94-105.

28. Alaoui-Jamali MA, Rossignol G, Castonguay A. Protective effect of vitamin A against the genotoxicity of NNK, a nicotine-derived N-nitrosamine. Carcinogenesis. 1991;12:379-84.

29. Stich HF, Rosin MP, Vallejera MO. Reduction with vitamin A and betacarotene administration of proportion of micronucleated buccal mucosal cells in Asian betel nut and tobacco chewers. Lancet. 1984;2:1204-6.

30. Stich HF, Mathew B, Sankaranaryana R, Nair MK. Remission of precancerous lesions in the oral cavity of tobacco chewers and maintenance of the protective effect of beta-carotene or vitamin A. Am J Clin Nutr. 1991;53(1suppl):298S-304S.

31. Toma S, Losardo PL, Vincent M and Palumbo R. Effectiveness of beta carotene in cancer chemoprevention. Eur J Cancer Prev. 1995;4:21323.

32. Shahin AA, Ismail MM, Saleh AM, Moustafa HA, Aboul-Ella AA, Gabr HM. Protective effect of folinic acid on low-dose methotrexate genotoxicity. Z Rheumatol. 2001;60:63-8.

33. Keshava C, Keshava N, Whong WZ, Nath J, Ong TM. Inhibition of methotrexate-induced chromosomal damage by folinic acid in V79 cells. Mut Res. 1998:397:221-8. 